\title{
Fuzzy Soft Gamma Semigroups
}

\author{
Muhammad Akram*, J. Kavikumar and Azme Bin Khamis \\ Department of Mathematics and Statistics, Faculty of Science, Technology and Human Development, University Tun Hussein Onn \\ Malaysia, Malaysia
}

Received: 28 Jun. 2013, Revised: 25 Sep. 2013, Accepted: 29 Sep. 2013

Published online: 1 Mar. 2014

\begin{abstract}
The aim of this paper is to apply the concept of fuzzy soft sets over a $\Gamma$-semigroup. Here the notion of fuzzy soft ideals over a $\Gamma$-semigroup has been introduced. The special union, intersection and product of fuzzy soft ideals over a $\Gamma$-semigroup have been defined and proved that these are also fuzzy soft $\Gamma$-ideals over the $\Gamma$-semigroup.
\end{abstract}

Keywords: $\Gamma$-semigroup, fuzzy soft set, fuzzy soft $\Gamma$-semigroup, fuzzy soft ideals.

\section{Introduction}

Zadeh [27] in 1965, introduced the basic concept of fuzzy sets, which became an imprtant part of research in Mathematics. Kuroki [10,11,12] presented the notion of fuzzy ideals and fuzzy bi-ideals in semigroups. He characterized several classes of semigroups in the terms of fuzzy ideals.

Sen and Saha [21] in 1986, introduced the notion of $\Gamma$-semigroup. They formed a relation between regular $\Gamma$-semigroup and $\Gamma$-group (see also $[16,17])$ Dutta and Adhikari [8] introduced prime ideals in $\Gamma$-semigroups. The concept of bi-ideals in $\Gamma$-semigroups was presented by Chinram and Jirojkul [7]. Shabir and Ali [24], studied prime bi-ideals in $\Gamma$-semigroups.

Sardar et al. $[19,20]$ gave the concept of fuzzy prime, semiprime ideals and also fuzzy ideal extension in $\Gamma$-semigroups. They also introduced the notions of fuzzy bi-ideals and fuzzy quasi-ideals in $\Gamma$-semigroups [20]. William et al. [25] also discussed fuzzy bi- $\Gamma$-ideals in $\Gamma$-semigroups. Faisal et al. [9], discussed the $\left(\in, \in \vee q_{k}\right)$-fuzzy $\Gamma$-ideals of $\Gamma$-semigroups.

Molodtsov [15] initiated the concept of soft set theory in 1999 and used this concept for the modeling of uncertainty. Maji et al. [13] defined some binary operations on soft sets, which were later corrected by Ali et al. [3] Shabir and Ali [23] introduced the notion of soft semigroups. The soft ternary semigroups were studied by Shabir and Ahmad [22]. Changphas and Thongkam [6] gave the notion of soft $\Gamma$-semigroups.
In 2001 Maji et al. [14] introduced the notion of fuzzy soft set as a combination of fuzzy set and soft set. They studied the union, intersection, compliment and De Morgan Law etc. for fuzzy soft sets. Ahmad and Kharal [1] improved the results of Maji et al. Aygunoglu and Aygun [4] extended Aktas and Cagman [2] soft groups concept for fuzzy soft groups. Yang [26] introduced the notion of fuzzy soft semigroups and fuzzy soft ideals. Recently, Bora et al. [5] defined some operations of fuzzy soft sets and explained them with examples.

The purpose of this paper is to extend the concepts of fuzzy soft sets to the theory of $\Gamma$-semigroups. Here, the notion of fuzzy soft left (right) ideals, fuzzy soft interior and fuzzy soft bi-ideals over a $\Gamma$-semigroup have been introduced. Also the characterization and algebraic properties of these ideals have been investigated.

\section{Preliminarie}

Let $S=\{x, y, z, \ldots\}$ and $\Gamma=\{\alpha, \beta, \gamma, \ldots\}$ be two non-empty sets. Then $S$ is called a $\Gamma$-semigroup if it satisfies

(i) $x \gamma y \in S$

(ii) $(x \beta y) \gamma z=x \beta(y \gamma z)$, for all $x, y, z \in S$ and $\beta, \gamma \in \Gamma$.

A non-empty subset $A$ of a $\Gamma$-semigroup $S$ is called a $\Gamma$-subsemigroup of $S$ if $A \Gamma A \subseteq A$. A left (right) $\Gamma$-ideal of a $\Gamma$-semigroup $S$ is a non-empty subset $A$ of $S$ such that $S \Gamma A \subseteq A(A \Gamma S \subseteq A)$ and a two sided $\Gamma$-ideal or simply a $\Gamma$-ideal is that which is both a left and a right $\Gamma$-ideal of

\footnotetext{
*Corresponding author e-mail: makram_69@yahoo.com
} 
$S$. A $\Gamma$-subsemigroup $B$ of a $\Gamma$-semigroup $S$ is called a bi$\Gamma$-ideal of $S$ if $B \Gamma S \Gamma B \subseteq B$. A $\Gamma$-subsemigroup $A$ of a $\Gamma$ semigroup $S$ is called an interior $\Gamma$-ideal of $S$ if $S \Gamma A \Gamma S \subseteq$ $A$. An ideal $I$ of a $\Gamma$-semigroup $S$ is called a prime $\Gamma$ ideal if for any ideals $A$ and $B$ of $S, A \Gamma B \subseteq I$ implies that $A \subseteq I$ or $B \subseteq I$ and is called semiprime $\Gamma$-ideal if $A \Gamma A \subseteq I$ implies that $A \subseteq I$. An element $x$ of a $\Gamma$-semigroup $S$ is called regular if there exist an element $s \in S$ and $\alpha, \beta \in \Gamma$ such that $x=x \alpha s \beta x$ and $S$ is called a regular $\Gamma$-semigroup if every element of $S$ is regular.

A fuzzy set $\mu$ in a non-empty set $X$ is a function, $\mu$ : $X \rightarrow[0,1]$ where the functions, $\mu: X \rightarrow[0,1]$ denotes the degree of membership of $x \in X$ in $[0,1]$. The compliment of $\mu$, denoted by $\bar{\mu}$ is the fuzzy set in $X$ given by $\bar{\mu}=$ $1-\mu(x)$ for all $x \in X$. The union and intersection of fuzzy sets is defined as

$$
\mu \cup v=\max \{\mu(x), v(x)\}, \text { for all } x \in X
$$

and $\mu \cap v=\min \{\mu(x), v(x)\}$, for all $x \in X$.

For any $t \in[0,1], A^{t}=\{x \in X \mid \mu(x) \geq t\}$. This is called the a $t$-level cut of $A$.

Definition 1.[15] Let $U$ be an initial universe set and $E$ be the set of parameters. Let $P(U)$ denotes the power set of $U$. A pair $(F, E)$ is called a soft set over $U$, where $F$ is a mapping given by, $F: E \rightarrow P(U)$.

Definition 2.[14] Let $U$ be an initial universe set and $E$ be the set of parameters. Let $A$ be a non empty subset of $E$ and $\mathscr{F}(U)$ be the collection of all fuzzy subsets of $U$ then the pair $(\widehat{f}, A)$ is called a fuzzy soft set (FSS) over $U$, where $\widehat{f}$ is a mapping given by, $\widehat{f}: A \rightarrow \mathscr{F}(U)$.

For each $a \in A$, we denote $\widehat{f}(a)$ by $f_{a}$, which is a fuzzy set over $U$.

Definition 3. [14] For any two fuzzy soft sets (FSS), ( $\widehat{f}, A)$ and $(\widehat{g}, B)$ over a common universe $U$, we say that $(\widehat{f}, A)$ is a fuzzy soft subset of $(\widehat{g}, B)$ if $A \subseteq B$ and $\widehat{f}(a) \subseteq \widehat{g}(a)$, for all $a \in A$. We write this as $(\widehat{f}, A) \widehat{\subseteq}(\widehat{g}, B)$.

Here $(\widehat{g}, B)$ is called fuzzy soft superset. $(\widehat{f}, A)$ and $(\widehat{g}, B)$ over a common universe $U$ are said to be fuzzy soft equal if, $(\widehat{f}, A) \widehat{\subseteq}(\widehat{g}, B)$ and $(\widehat{g}, B) \widehat{\subseteq}(\widehat{f}, A)$.

Definition 4.[14] Let $(\widehat{f}, A)$ and $(\widehat{g}, B)$ be two fuzzy soft sets over a common universe $U$ then " $(\widehat{f}, A) A N D(\widehat{g}, B)$ ", denoted by $(\widehat{f}, A) \widehat{\wedge}(\widehat{g}, B)$ is defined as $(\widehat{f}, A) \widehat{\wedge}(\widehat{g}, B)=$ $(\widehat{h}, C)$, where $C=A \times B$ and $\widehat{h}(a, b)=\widehat{f}(a) \cap \widehat{g}(b)$, for all $(a, b) \in C=A \times B$.

Definition 5.[14] Let $(\widehat{f}, A)$ and $(\widehat{g}, B)$ be two fuzzy soft sets over a common universe $U$ then " $(\widehat{f}, A)$ OR $(\widehat{g}, B)$ ", denoted by $(\widehat{f}, A) \widehat{V}(\widehat{g}, B)$ is defined as $(\widehat{f}, A) \widehat{V}(\widehat{g}, B)=$ $(\widehat{k}, C)$, where $C=A \times B$ and $\widehat{k}(a, b)=\widehat{f}(a) \cup \widehat{g}(b)$, for all $(a, b) \in C=A \times B$.
Definition 6. [14] Let $(\widehat{f}, A)$ and $(\widehat{g}, B)$ be two fuzzy soft sets over a common universe $U$ then their union is a fuzzy soft set over $U$ denoted by $(\widehat{f}, A) \widehat{\cup}(\widehat{g}, B)$ and is defined as $(\widehat{f}, A) \widehat{\cup}(\widehat{g}, B)=(\widehat{h}, C)$, where $C=A \cup B$ and

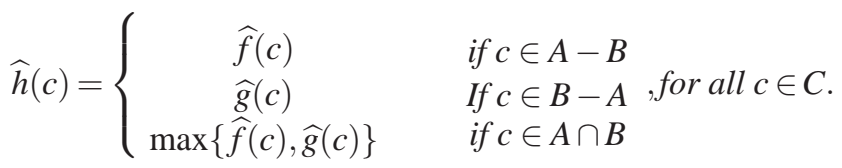

Definition 7.[14] Let $(\widehat{f}, A)$ and $(\widehat{g}, B)$ be two fuzzy soft sets over a common universe $U$ then their intersection is a fuzzy soft set over $U$ denoted by $(\widehat{f}, A) \widehat{\cap}(\widehat{g}, B)$ and is defined as $(\widehat{f}, A) \widehat{\cap}(\widehat{g}, B)=(\widehat{h}, C)$, where $C=A \cup B$ and

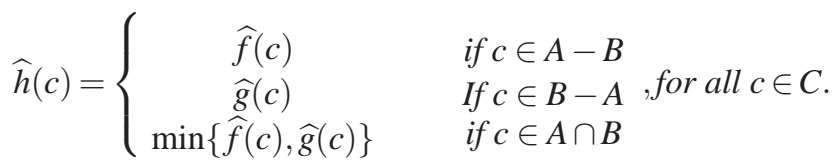

Except above definitions of union and intersection of fuzzy soft sets, we may some times use another definitions of union and intersection given as follows.

Definition 8.Let $(\widehat{f}, A)$ and $(\widehat{g}, B)$ be two fuzzy soft sets over a common universe $U$ such that $A \cap B \neq \phi$. The biunion of $(\widehat{f}, A)$ and $(\widehat{g}, B)$ is defined to be a fuzzy soft set $(\widehat{h}, C)$ over $U$, where $C=A \cap B$ and $\widehat{h}(c)=\widehat{f}(c) \cup \widehat{g}(c)$ for all $c \in C$. This is denoted by $(\widehat{h}, C)=(\widehat{f}, A) \widehat{\sqcup}(\widehat{g}, B)$.

Definition 9.Let $(\widehat{f}, A)$ and $(\widehat{g}, B)$ be two fuzzy soft sets over a common universe $U$ such that $A \cap B \neq \phi$. The biintersection of $(\widehat{f}, A)$ and $(\widehat{g}, B)$ is defined to be a fuzzy soft set $(\widehat{h}, C)$ over $U$, where $C=A \cap B$ and $\widehat{h}(c)=\widehat{f}(c) \cap$ $\widehat{g}(c)$ for all $c \in C$. This is denoted by $(\widehat{h}, C)=(\widehat{f}, A) \widehat{\Pi}$ $(\widehat{g}, B)$.

If $\left\{\left(\widehat{f}_{i}, A_{i}\right): i \in I\right\}$ be a collection of fuzzy soft sets over a common universe $U$ such that $\cap_{i \in I} A_{i} \neq \phi$ then similarly,

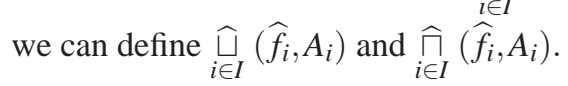

\section{Fuzzy soft ideals over Gamma semigroup}

In what follows, let $S$ denotes a $\Gamma$-semigroup unless otherwise specified.

Definition 10.Let $(\widehat{\mu}, A)$ be a fuzzy soft set over a $\Gamma$-semigroup $S$, then $(\widehat{\mu}, A)$ is called a fuzzy soft $\Gamma$-subsemigroup over $S$ if

$$
\mu_{a}(x \gamma y) \geq \min \left\{\mu_{a}(x), \mu_{a}(y)\right\}
$$

for all $a \in A, x, y, \in S$ and $\gamma \in \Gamma$. 
Definition 11.Let $(\widehat{\mu}, A)$ be a fuzzy soft set over a $\Gamma$-semigroup $S$, then $(\widehat{\mu}, A)$ is called a fuzzy soft left (right) $\Gamma$-ideal over $S$ if

$$
\mu_{a}(x \gamma y) \geq \mu_{a}(y) \quad\left(\mu_{a}(x \gamma y) \geq \mu_{a}(x)\right)
$$

for all $a \in A, x, y \in S$ and $\gamma \in \Gamma$.

Definition 12.A fuzzy soft set $(\widehat{\mu}, A)$ over a $\Gamma$-semigroup $S$ is called a fuzzy soft $\Gamma$-ideal over $S$ if and only if it is both a fuzzy soft left and a fuzzy soft right $\Gamma$-ideal over $S$. Equivalently, we can define as,

Definition 13.A fuzzy soft set $(\widehat{\mu}, A)$ over a $\Gamma$-semigroup $S$ is called a fuzzy soft $\Gamma$-ideal over $S$ if

$$
\mu_{a}(x \gamma y) \geq \max \left\{\mu_{a}(x), \mu_{a}(y)\right\} .
$$

It is clear that any fuzzy soft left (right) $\Gamma$-ideal over $S$ is a fuzzy soft $\Gamma$-subsemigroup of $S$ but the converse is not true.

Example 1.Let $S=\{a, b, c\}, \Gamma=\{\gamma\}$ then $S$ is a $\Gamma$-semigroup under the operation defined in the table,

$\begin{array}{llll}\gamma & a & b & c \\ a & a & c & c \\ b & c & b & c \\ c & c & c & c\end{array}$

Let $E=\{u, v, w\}, A=\{u, w\}$ then $(\widehat{\mu}, A)$ is a fuzzy soft set defined as, $\mu_{u}=\{(a, 0.1),(b, 0.3),(c, 0.5)\}, \mu_{w}=$ $\{(a, 0.2),(b, 0.4),(c, 0.8)\}$. It is easy to verify that $(\widehat{\mu}, A)$ is a fuzzy soft left and a fuzzy soft right $\Gamma$-ideal over $S$. Hence $(\widehat{\mu}, A)$ is a fuzzy soft $\Gamma$-ideal over $S$.

Let $B=\{v\}$ and $\lambda_{v}=\{(a, 0.1),(b, 0.8),(c, 0.3)\}$ then $(\widehat{\lambda}, B)$ is a fuzzy soft $\Gamma$-subsemigroup but it is not a soft $\Gamma$-ideal over $S$.

Definition 14. A fuzzy soft $\Gamma$-subsemigroup $(\widehat{\mu}, A)$ of $S$ is called a fuzzy soft interior $\Gamma$-ideal over $S$ if

$\mu_{a}(x \alpha z \beta y) \geq \mu_{a}(z)$ for all $, x, y, z, \in S, \alpha, \beta \in \Gamma$ and $a \in A$.

Definition 15.A fuzzy soft $\Gamma$-subsemigroup $(\widehat{\mu}, A)$ of $S$ is called a fuzzy soft $\Gamma$-bi-ideal over $S$ if

$\mu_{a}(x \alpha z \beta y) \geq \min \left\{\mu_{a}(x), \mu_{a}(y)\right\}$ for all $, x, y, z \in S, \alpha, \beta \in \Gamma$ and $a \in A$.

Lemma 1.A fuzzy soft set $(\widehat{\mu}, A)$ over a $\Gamma$-semigroup $S$ is a fuzzy soft ideal over $S$ if and only if $\widehat{\mu}(a)^{t}=\mu_{a}^{t}$ is an ideal of $S$ for all $t \in[0,1]$ and $a \in A$.

Proof.Straightforward.

Lemma 2.Let $(\widehat{\mu}, A)$ be a fuzzy soft ideal over a $\Gamma$-semigroup $S$. For any non-null, $B \subset A,(\widehat{\mu}, B)$ is also a fuzzy soft ideal over $S$.

Proof.Straightforward.
Theorem 1.Let $(\widehat{\mu}, A)$ and $(\widehat{v}, B)$ be two fuzzy soft ideals (left, right) over a $\Gamma$-semigroup $S$. Then $(\widehat{\mu}, A) \widehat{\wedge}(\widehat{v}, B)$ and $(\widehat{\mu}, A) \widehat{\Pi}(\widehat{v}, B)$ are also fuzzy soft ideals (left, right) over $S$.

Proof.Let $(\widehat{\mu}, A)$ and $(\widehat{v}, B)$ be two fuzzy soft ideals (left , right) over a $\Gamma$-semigroup $S$ then as definede $(\widehat{\mu}, A)$ $\widehat{\wedge}(\widehat{v}, B)=(\widehat{\lambda}, C)$, where $C=A \times B$ and $\widehat{\lambda}(a, b)=\widehat{\mu}(a) \cap$ $\widehat{v}(b)$, for all $(a, b) \in C=A \times B$. As $(\widehat{\mu}, A)$ and $(\widehat{v}, B)$ are fuzzy soft ideals (left, right) over $S$ then for $(a, b) \in C=$ $A \times B$, we have

$\widehat{\lambda}(a, b)(x \gamma y)=\lambda_{(a, b)}(x \gamma y)=\left(\mu_{a} \cap v_{b}\right)(x \gamma y)=$ $\min \left\{\mu_{a}(x \gamma y), v_{b}(x \gamma y)\right\}$

$\geq \min \left\{\max \left\{\mu_{a}(x), \mu_{a}(y)\right\}, \max \left\{v_{b}(x), v_{b}(y)\right\}\right\}$

$=\max \left\{\min \left\{\mu_{a}(x), v_{b}(x)\right\}, \min \left\{\mu_{a}(y), v_{b}(y)\right\}\right\}$

$\left.\left.=\max \left\{\left(\mu_{a} \cap v_{b}\right)(x)\right),\left(\mu_{a} \cap v_{b}\right)(y)\right)\right\}$

$=\max \left\{\lambda_{(a, b)}(x), \lambda_{(a, b)}(y)\right\}$

$=\max \{\widehat{\lambda}(a, b)(x), \widehat{\lambda}(a, b)(y)\}$, for all $x, y \in S, \gamma \in \Gamma$ and $(a, b) \in C=A \times B$. Which implies that $(\widehat{\mu}, A)$ $\widehat{\wedge}(\widehat{v}, B)=(\widehat{\lambda}, C)$ is a fuzzy soft ideal (left, right) over $S$.

Similarly, we can prove that $(\widehat{\mu}, A) \widehat{\Pi}(\widehat{v}, B)$ is also fuzzy soft ideal (left, right) over $S$.

Theorem 2.Let $(\widehat{\mu}, A)$ and $(\widehat{v}, B)$ be two fuzzy soft bi-ideals (interior) over a $\Gamma$-semigroup $S$ then $(\widehat{\mu}, A)$ $\widehat{\wedge}(\widehat{v}, B)$ and $(\widehat{\mu}, A) \widehat{\Pi}(\widehat{v}, B)$ are also fuzzy soft bi-ideals (interior) over $S$.

Proof.As $(\widehat{\mu}, A)$ and $(\widehat{v}, B)$ are fuzzy soft bi-ideals (interior) over $S$ then they are also fuzzy soft ideals over $S$ and by Theorem $1,(\widehat{\mu}, A) \widehat{\wedge}(\widehat{v}, B)$ and $(\widehat{\mu}, A) \widehat{\Pi}(\widehat{v}, B)$ are also fuzzy soft ideals and hence $\Gamma$-subsemigroup of $S$. Since, $(\widehat{\mu}, A) \widehat{\wedge}(\widehat{v}, B)=(\widehat{\lambda}, C)$, where $C=A \times B$ and $\widehat{\lambda}(a, b)=\widehat{\mu}(a) \cap \widehat{v}(b)$, for all $(a, b) \in C=A \times B$. Let $x, y, z \quad \in \quad S, \quad \alpha, \beta \quad \in \quad \Gamma \quad$ then $\widehat{\lambda}(a, b)(x \alpha z \beta y)=\lambda_{(a, b)}(x \alpha z \beta y)=\left(\mu_{a} \cap v_{b}\right)(x \alpha z \beta y)=$ $\min \left\{\mu_{a}(x \alpha z \beta y), v_{b}(x \alpha z \beta y)\right\}$

$$
\begin{aligned}
& \geq \min \left\{\min \left\{\mu_{a}(x), \mu_{a}(y)\right\}, \min \left\{v_{b}(x), v_{b}(y)\right\}\right. \\
& =\min \left\{\min \left\{\mu_{a}(x), v_{b}(x)\right\}, \min \left\{\mu_{a}(y), v_{b}(y)\right\}\right\} \\
& \left.\left.=\min \left\{\left(\mu_{a} \cap v_{b}\right)(x)\right),\left(\mu_{a} \cap v_{b}\right)(y)\right)\right\} \\
& =\min \left\{\lambda(a, b)(x), \lambda{ }_{(a, b)}(y)\right\} \\
& =\min \{\widehat{\lambda}(a, b)(x), \hat{\lambda}(a, b)(y)\} .
\end{aligned}
$$

Hence $(\widehat{\mu}, A) \widehat{\wedge}(\widehat{v}, B)=(\widehat{\lambda}, C)$ is an fuzzy soft bi-ideal (interior) over $S$.

Similarly, we can prove that $(\widehat{\mu}, A) \widehat{\Pi}(\widehat{v}, B)$ is also fuzzy soft bi-ideal (interior) over $S$.

Theorem 3.Let $(\widehat{\mu}, A)$ and $(\widehat{v}, B)$ be two fuzzy soft ideals (left, right) over a $\Gamma$-semigroup $S$ then $(\widehat{\mu}, A) \widehat{V}(\widehat{v}, B)$ and $(\widehat{\mu}, A) \widehat{\sqcup}(\widehat{v}, B)$ are also fuzzy soft ideals (left, right) over $S$.

Proof.Let $(\widehat{\mu}, A)$ and $(\widehat{v}, B)$ be two fuzzy soft ideals (left, right) over a $\Gamma$-semigroup $S$. Then $(\widehat{\mu}, A) \widehat{V}(\widehat{v}, B)$ is defined as $(\widehat{\mu}, A) \widehat{V}(\widehat{v}, B)=(\widehat{\delta}, C)$, where $C=A \times B$ and $\widehat{\delta}(a, b)=\widehat{\mu}(a) \cup \widehat{v}(b)$, for all $(a, b) \in C=A \times B$. As 
$(\widehat{\mu}, A)$ and $(\widehat{v}, B)$ are fuzzy soft ideals (left, right) over $S$, so we have for all $x, y, z \in S$ and $\gamma \in \Gamma$,

$\widehat{\delta}(a, b)(x \gamma y)=\delta_{(a, b)}(x \gamma y)=\left(\mu_{a} \cup v_{b}\right)(x \gamma y)=$ $\max \left\{\mu_{a}(x \gamma y), v_{b}(x \gamma y)\right\}$

$\geq \max \left\{\max \left\{\mu_{a}(x), \mu_{a}(y)\right\}, \max \left\{v_{b}(x), v_{b}(y)\right\}\right\}$

$=\max \left\{\max \left\{\mu_{a}(x), v_{b}(x)\right\}, \max \left\{\mu_{a}(y), v_{b}(y)\right\}\right\}$

$=\max \left\{\left(\mu_{a} \cup v_{b}\right)(x),\left(\mu_{a} \cup v_{b}\right)(y)\right\}$

$=\max \left\{\boldsymbol{\delta}_{(a, b)}(x), \boldsymbol{\delta}_{(a, b)}(y)\right\}$

$=\max \{\widehat{\delta}(a, b)(x), \widehat{\delta}(a, b)(y)\}$.

Which implies that $(\widehat{\mu}, A) \widehat{V}(\widehat{v}, B)=(\widehat{\delta}, C)$ is a fuzzy soft ideals (left, right) over $S$.

Similarly, we can prove that $(\widehat{\mu}, A) \widehat{\sqcup}(\widehat{v}, B)$ is also a fuzzy soft ideal (left, right) over $S$.

Theorem 4.Let $(\widehat{\mu}, A)$ and $(\widehat{v}, B)$ be two fuzzy soft bi-ideals (interior) over a $\Gamma$-semigroup $S$ then $(\widehat{\mu}, A) \widehat{\vee}(\widehat{v}, B)$ and $(\widehat{\mu}, A) \widehat{\sqcup}(\widehat{v}, B)$ are also fuzzy soft bi-ideal (interior) over $S$.

Proof.Straightforward.

Theorem 5.Let $(\widehat{\mu}, A)$ and $(\widehat{v}, B)$ be two fuzzy soft ideals (left, right) over a $\Gamma$-semigroup $S$ then $(\widehat{\mu}, A) \widehat{\cap}(\widehat{v}, B)$ and $(\widehat{\mu}, A) \widehat{\cup}(\widehat{v}, B)$ are also fuzzy soft ideals (left, right) over $S$.

Proof.Let $(\widehat{\mu}, A)$ and $(\widehat{v}, B)$ be two fuzzy soft ideals (left, right) over a $\Gamma$-semigroup $S$ then $(\widehat{\mu}, A) \widehat{\cap}(\widehat{v}, B)=(\widehat{\lambda}, C)$, where $C=A \cup B$ and

$$
\widehat{\lambda}(c)=\left\{\begin{array}{ll}
\widehat{\mu}(c) & \text { if } c \in A-B \\
\widehat{v}(c) & \text { if } c \in B-A \\
\min \{\widehat{\mu}(c), \widehat{v}(c)\} & \text { if } c \in A \cap B
\end{array} \text {, for all } c \in\right.
$$

C.

Let $c \in C$ and $x, y \in S$ and $\gamma \in \Gamma$ then we have,

(i) If $c \in A-B$, then

$\widehat{\lambda}(c)(x \gamma y)=\widehat{\mu}(c)(x \gamma y)=\mu_{c}(x \gamma y) \geq$ $\max \left\{\mu_{c}(x), \mu_{c}(y)=\max \{\widehat{\mu}(c)(x), \widehat{\mu}(c)(y)\}\right.$

$=\max \{\widehat{\lambda}(c)(x), \widehat{\lambda}(c)(y)\}$

(ii) If $c \in B-A$, then

$\widehat{\lambda}(c)(x \gamma y)=\widehat{v}(c)(x \gamma y)=v_{c}(x \gamma y) \geq$ $\max \left\{\boldsymbol{v}_{c}(x), \boldsymbol{v}_{c}(y)\right\}=\max \{\widehat{\boldsymbol{v}}(c)(x), \widehat{\boldsymbol{v}}(c)(y)\}$

$=\max \{\widehat{\lambda}(c)(x), \widehat{\lambda}(c)(y)\}$

(iii) If $c \in A \cap B, \quad$ then $\widehat{\lambda}(c)=\min \{\widehat{\mu}(c), \widehat{v}(c)\}=\widehat{\mu}(c) \cap \widehat{v}(c)$.

We can easily verify that $\widehat{\lambda}(c)(x \gamma y) \geq \max \{\widehat{\lambda}(c)(x), \widehat{\lambda}(c)(y)\}$.

Hence, for all $c \in C$ and $x, y \in S$ and $\gamma \in \Gamma$, we can write $\widehat{\lambda}(c)(x \gamma y) \geq \max \{\widehat{\lambda}(c)(x), \widehat{\lambda}(c)(y)\}$.

Which shows that $(\widehat{\mu}, A) \widehat{\cap}(\widehat{v}, B)=(\widehat{\lambda}, C)$ is a fuzzy soft ideal (left, right) over $S$.

Similarly, we can prove that $(\widehat{\mu}, A) \widehat{\cup}(\widehat{v}, B)$ is also a fuzzy soft ideal (left, right) over $S$.

Theorem 6.Let $(\widehat{\mu}, A)$ and $(\widehat{v}, B)$ be two fuzzy soft bi-ideals (interior) over a $\Gamma$-semigroup $S$ then $(\widehat{\mu}, A) \widehat{\cap}(\widehat{v}, B)$ and $(\widehat{\mu}, A) \widehat{\cup}(\widehat{v}, B)$ are also fuzzy soft bi-ideals (interior) over $S$.
Proof.Straightforward.

Theorem 7.Let $\Delta(S, E)$, be the collection of all fuzzy soft ideals (left, right, interior, bi) over a $\Gamma$-semigroup $S$. Then $(\Delta(S, E), \widehat{\cup}, \widehat{\Pi})$ is a complete distributive lattice under the relation $\widehat{\subseteq}$.

Proof.Let $(\widehat{\mu}, A)$ and $(\widehat{v}, B)$ be two fuzzy soft ideals (left, right, interior, bi) over a $\Gamma$-semigroup $S$ that is $(\widehat{\mu}, A),(\widehat{v}, B) \in \Delta(S, E)$ then as, we proved above, $(\widehat{\mu}, A) \widehat{\cup}(\widehat{v}, B)$ and $(\widehat{\mu}, A) \widehat{\Pi}(\widehat{v}, B)$ are fuzzy soft ideals (left right, interior, bi) over $S$, implies that $(\widehat{\mu}, A) \widehat{\cup}(\widehat{v}, B),(\widehat{\mu}, A) \widehat{\Pi}(\widehat{v}, B) \in \Delta(S, E)$. Obviously, we can say that $(\hat{\mu}, A) \widehat{\cup}(\widehat{v}, B)$ is the least upper bound and $(\widehat{\mu}, A) \widehat{\Pi}(\widehat{v}, B)$ is the greatest lower bound of the subclass $\{(\widehat{\mu}, A),(\widehat{v}, B)\}$. Hence for any arbitrary collection of $\Delta(S, E)$, there exist a least upper bound and a greatest lower bound, which implies that $\Delta(S, E)$ is a complete lattice.

Now, for $(\widehat{\mu}, A),(\widehat{v}, B)$ and $(\widehat{\eta}, C) \in \Delta(S, E)$, we have

$$
(\widehat{\mu}, A) \widehat{\Pi}((\widehat{v}, B) \widehat{\cup}(\widehat{\eta}, C))=(\widehat{\delta}, A \cap(B \cup C)) .
$$

Also $((\widehat{\mu}, A) \widehat{\Pi}(\widehat{v}, B) \widehat{\cup}((\widehat{\mu}, A) \widehat{\Pi}(\widehat{\eta}, C))=(\widehat{\omega},(A \cap B) \cup(A \cap C))$

$$
=(\widehat{\omega}, A \cap(B \cup C))
$$

Easily, we can show that for any $z \in A \cap(B \cup C), \widehat{\delta}(z)=$ $\widehat{\omega}(z)$, which implies that

$(\widehat{\mu}, A) \widehat{\Pi}((\widehat{v}, B) \widehat{\cup}(\widehat{\eta}, C))=((\widehat{\mu}, A) \widehat{\Pi}(\widehat{v}, B) \widehat{\cup}((\widehat{\mu}, A) \widehat{\Pi}(\widehat{\eta}, C))$

Hence $\Delta(S, E)$ is a complete distributive lattice.

Theorem 8.Let $\Delta(S, E)$, be the collection of all fuzzy soft ideals (left, right, interior, bi) over a $\Gamma$-semigroup $S$. Then $(\Delta(S, E), \widehat{\sqcup}, \widehat{\cap})$ is a complete distributive lattice under the relation $\widehat{\subseteq}^{\prime}$.

Proof.Straightforward.

Now, let $D \subseteq E$ be a specific family of parameters. let the set of fuzzy soft ideals over a $\Gamma$-semigroup $S$ with parameter set $D$ is denoted by $\Delta_{D}(S)$, where $\Delta_{D}(S)=\{(\widehat{\mu}, A) \in \Delta(S, E) \mid \widehat{\mu}: D \rightarrow P(F S(S)\}$.

Lemma 3.Let $(\widehat{\mu}, A)$ and $(\widehat{v}, B) \in \Delta_{D}(S)$, then $(\widehat{\mu}, A) \widehat{\cup}(\widehat{v}, B) \in \Delta_{D}(S)$ and $(\widehat{\mu}, A) \widehat{\sqcap}(\widehat{v}, B) \in \Delta_{D}(S)$.

Proof.Straightforward.

Lemma 4.Let $(\widehat{\mu}, A)$ and $(\widehat{v}, B) \in \Delta_{D}(S)$, then $(\widehat{\mu}, A) \widehat{\cap}(\widehat{v}, B) \in \Delta_{D}(S)$ and $(\widehat{\mu}, A) \widehat{\sqcup}(\widehat{v}, B) \in \Delta_{D}(S)$.

Proof.Straightforward.

Theorem 9. $\left(\Delta_{D}(S), \widehat{\Pi}, \widehat{\cup}\right)$ is a sublattice of $(\Delta(S, E), \widehat{\Pi}, \widehat{\cup})$ and $\left(\Delta_{D}(S), \widehat{\sqcup}, \widehat{\cap}\right)$ is a sublattice of $(\Delta(S, E), \widehat{\sqcup}, \widehat{\cap})$.

Proof.Straightfoeward.

Definition 16.Let $(\widehat{\mu}, A)$ and $(\widehat{v}, B)$ be two fuzzy soft sets over a $\Gamma$-semigroup $S$. Then their product is defined as $(\widehat{\mu}, A) \circ(\widehat{v}, B)=(\widehat{\mu} \Gamma \widehat{v}, C)$, where $C=A \cup B$ and 


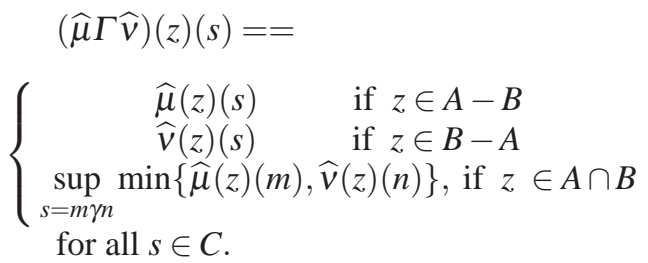

Theorem 10.Let $(\widehat{\mu}, A)$ and $(\widehat{v}, B)$ be two fuzzy soft ideals (left, right, interior, bi) over a $\Gamma$-semigroup $S$ then their product $(\widehat{\mu}, A) \circ(\widehat{v}, B)$ is also a fuzzy soft ideal (left, right, interior, bi) over $S$.

Proof.Let $(\widehat{\mu}, A)$ and $(\widehat{v}, B)$ be two fuzzy soft ideals over a $\Gamma$-semigroup $S$. Let $z \in C=A \cup B, x, y \in S$ and $\gamma \in \Gamma$. We have,

(i) $z \in A-B$, then

$$
\begin{aligned}
(\widehat{\mu} \Gamma \widehat{v})(z)(x \gamma y) & =\widehat{\mu}(z)(x \gamma y) \\
& \geq \max \{\widehat{\mu}(z)(x), \widehat{\mu}(z)(y)\} \\
& =\max \{(\widehat{\mu} \Gamma \widehat{v})(z)(x),(\widehat{\mu} \Gamma \widehat{v})(z)(y)\} .
\end{aligned}
$$

(ii) $z \in B-A$, same as proved in (i).

(iv) $z \in A \cap B$, then

$$
\begin{aligned}
(\widehat{\mu} \Gamma \widehat{v})(z)(x) & =\sup _{x=m \gamma n} \min \{(\widehat{\mu})(z)(m), \widehat{v}(z)(n)\} \\
\leq & \sup _{x \alpha y=u \gamma v \alpha y} \min \{(\widehat{\mu})(z)(m), \widehat{v}(z)(n)\} \\
& \leq \sup _{x \alpha y=u \gamma w}\{\min \{(\widehat{\mu})(z)(m), \widehat{v}(z)(w)\}\}=
\end{aligned}
$$

$(\widehat{\mu} \Gamma \widehat{v})(z)(x \alpha y)$

$\Rightarrow(\widehat{\mu} \Gamma \widehat{v})(z)(x) \leq(\widehat{\mu} \Gamma \widehat{v})(z)(x \alpha y)$. Similarly, we can show that,

$(\widehat{\mu} \Gamma \widehat{v})(z)(y) \leq(\widehat{\mu} \Gamma \widehat{v})(z)(x \alpha y)$.

Which implies that,

$\max \{(\widehat{\mu} \Gamma \widehat{v})(z)(x),(\widehat{\mu} \Gamma \widehat{v})(z)(y)\}$.

$(\widehat{\mu} \Gamma \widehat{v})(z)(x \alpha y) \geq$

Hence, $(\widehat{\mu}, A) \circ(\widehat{v}, B)$ is a fuzzy soft ideal over $S$.

Theorem 11.Let $S$ be a $\Gamma$-semigroup with identity $e$ and $\Omega(S, E)$ be the collection of all fuzzy soft ideals over $S$ with the property that $(\widehat{\mu}, A) \in \Omega(S, E)$ if and only if, $\widehat{\mu}(z)(e)=$ 1 then $(\Omega(S, E), \circ, \widehat{\Pi})$ is a complete lattice under $\widehat{\subseteq}$.

Proof.Let $(\widehat{\mu}, A)$ and $(\widehat{v}, B) \in \Omega(S, E)$ then $\widehat{\mu}(z)(e)=\widehat{v}(z)(e)=1$. As $(\widehat{\mu}, A)$ and $(\widehat{v}, B)$ be fuzzy soft ideals over $S$ then so is $(\widehat{\mu}, A) \widehat{\Pi}(\widehat{v}, B)$ and $(\widehat{\mu}, A) \circ(\widehat{v}, B)$ by Theorem 1 and Theorem 10. Also $(\widehat{\mu} \cap \widehat{v})(z)(e)=1$ and $(\widehat{\mu} \Gamma \widehat{v})(z)(e)=1$. Which implies that $(\widehat{\mu}, A) \widehat{\Pi}(\widehat{v}, B)$ and $(\widehat{\mu}, A) \circ(\widehat{v}, B) \in \Omega(S, E)$. Note that $(\widehat{\mu}, A) \widehat{\Pi}(\widehat{v}, B)$ is the greatest lower bound of the class $\{(\widehat{\mu}, A),(\widehat{v}, B)\}$. Now for least upper bound, let $z \in A \cup B$ and $x \in S$ then, we have

(i) If $z \in A-B$ then by definition, $(\widehat{\mu} \Gamma \widehat{v})(z)(x)=\widehat{\mu}(z)(x)$

(ii) If $z \in B-A$, then $(\widehat{\mu} \Gamma \widehat{v})(z)(x)=\widehat{v}(z)(x)$.

(iii) If $z \in A \cap B$, then as $e$ is identity in $S$, so

$$
\begin{aligned}
(\widehat{\mu} \Gamma \widehat{v})(z)(x) & =\sup _{x=x \gamma e}\{\min \{\widehat{\mu}(z)(x), \widehat{v}(z)(e)\}\} \\
& \geq \min \{\widehat{\mu}(z)(x), \widehat{v}(z)(e)\} \\
& =\widehat{\mu}(z)(x), \text { since } \widehat{v}(z)(e)=1 .
\end{aligned}
$$

Which implies that $(\widehat{\mu}, A) \widehat{\subseteq}(\widehat{\mu}, A) \circ(\widehat{v}, B)$. Similarly, we can show that $(\widehat{v}, B) \widehat{\subseteq}(\widehat{\mu}, A) \circ(\widehat{v}, B)$ implies that $(\widehat{\mu}, A) \circ(\widehat{v}, B)$ is an upper bound of $\{(\widehat{\mu}, A),(\widehat{v}, B)\}$. Now, let $(\widehat{\rho}, \Sigma) \in \Omega(S, E)$ such that $(\widehat{\mu}, A) \widehat{\widehat{\subseteq}}(\widehat{\rho}, \Sigma)$ and $(\widehat{v}, B) \widehat{\subseteq}(\widehat{\rho}, \Sigma)$.

Then $(\widehat{\mu}, A) \circ(\widehat{v}, B) \subseteq(\widehat{\rho}, \Sigma) \circ(\widehat{\rho}, \Sigma) \subseteq(\widehat{\rho}, \Sigma)$. Hence $(\widehat{\mu}, A) \circ(\widehat{v}, B)$ is the least upper bound of the class $\{(\widehat{\mu}, A),(\widehat{v}, B)\}$, which is an orbitrary subclass of $\Omega(S, E)$. Hence $(\Omega(S, E), \circ, \widehat{\Pi})$ is a complete lattice.

\section{References}

[1] B. Ahmad and A. Kharal, On fuzzy soft sets, Advances in fuzzy systems, 2009, 1-6 (2009).

[2] H. Aktaş and N. Cağman, Soft stes and soft groups, Inform. Sci., 177, 2726-2735 (2007).

[3] M. I. Ali, F. Feng, X. Y. Liu and M. Shabir, On some new operations in soft set theory, Comput. Math. Appl., 57, 15471553 (2009).

[4] A. Aygúnoğlu and H. Aygún, Introduction to fuzzy soft groups, Comput. Math. appl., 58, 1279-1286 (2009).

[5] M. Bora, T. J. Neog and D. K. Sut, A study on some operations of fuzzy soft sets, (Accepetd for IJMER).

[6] T. Changphas and B. Thongkam, On $\Gamma$-soft semigroups, Annals of Fuzzy Mathematics and Informatics, 4, 217-223 (2012).

[7] R. Chinram and C. Jirojkul, On bi- $\Gamma$-ideals in $\Gamma$-semigroups, Songklanakarin J. Sci. Technol., 29, 231-234 (2007).

[8] T. K. Dutta and N. C. Adhikari, On prime radical of $\Gamma$ semigroup, Bull. Cal. Math. Soc., 86, 437-444 (1994).

[9] Faisal, N. Yaqoob and R. Chinram, A note on $\left(\in, \in \vee q_{k}\right)$ fuzzy $\Gamma$-ideals of $\Gamma$-semigroups, Applied Mathematical Sciences, 6, 5013-5028 (2012).

[10] N. Kuroki, On fuzzy ideals and fuzzy bi-ideals in semigroups, Fuzzy sets and systems, 5203-215 (1981).

[11] N. Kuroki, Fuzzy semiprime ideals in semigroups, Fuzzy sets and systems, 8, 71-79 (1982).

[12] N. Kuroki, On fuzzy semigroups, Information Sciences, 53, 203-236 (1991).

[13] P. K. Maji, A. R. Roy and R.Biswas, Soft set theory, Comput. Math. Appl., 45, 555-562 (2003).

[14] P. K. Maji, A. R. Roy and R.Biswas, Fuzzy soft sets, The journal of fuzzy mathematics, 9, 589-602 (2001).

[15] D. Molodtsov, Soft set theory-First results, Comput. Math. Appl., 37, 19-31 (1999).

[16] N. K. Saha, On $\Gamma$-semigroup II, Bull. Calcutta Math. Soc., 79, 331-335 (1987).

[17] N. K. Saha, On $\Gamma$-semigroup III, Bull. Calcutta Math. Soc., 80, 1-13 (1988).

[18] S. K. Sardar and S. K. Majumder, On fuzzy ideals in $\Gamma$ semigroups, Int. J. Algebra, 3, 775-784 (2009).

[19] S. K. Sardar and S. K. Majumder, A note on characterization of prime ideals of $\Gamma$-semigroups in terms of fuzzy subsets, Int. J. Contemp. Math. Sci., 4, 1465-1472 (2009).

[20] S. K. Sardar, S. K. Majumder and Soumitra Kayal, On fuzzy bi-ideals and fuzzy quasi ideals in gamma-semigroups, arXiv:1101.4720v1 [math.GM], (2011).

[21] M. K. Sen and N.K. Saha, On $\Gamma$-semigroup I, Bull. Calcutta Math. Soc., 78, 180-186 (1986). 
[22] M. Shabir and A. Ahmad, On soft ternary semigroups, Annals of Fuzzy Math. Inform., 3, 39-59 (2012).

[23] M. Shabir and M. I. Ali, Soft ideals and generalized fuzzy ideals in semigroups, New Math. and Nat. Comput., 5, 599615 (2009).

[24] M. Shabir and S. Ali, Prime bi-ideals of $\Gamma$-semigroups, J. Adv. Res. in Pure Math., 4, 47-58 (2012).

[25] D. R. P. William, K. B. Latha and E. Chandrasekeran, Fuzzy bi- $\Gamma$-ideals in $\Gamma$-semigroups, Hacettepe Journal of Mathematics and statistics, 38, 1-15 (2009).

[26] C. F. Yang, Fuzzy soft semigroups and fuzzy soft ideals, Computers and Mathematics with Applications, 61, 255-261 (2011).

[27] L. A. Zadeh, Fuzzy sets, Information and Control, 8, 338353 (1965).

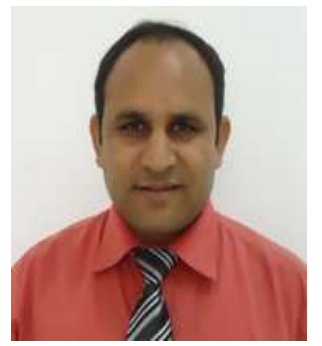

Muhammad Akram did his M.Sc with distinction from the "University of The Punjab", Lahore and M.Phil from Quaid-i-Azam University, Islamabad, Pakistan. Since 2008 he is a permanent faculty member at the "University of Gujrat", Gujrat, Pakistan. He is a Ph.D research scholar at the "Universiti Tun Hussein Onn Malaysia" Malaysia. His research interests are algebraic structures, fuzzy and intuitionistic fuzzy algebraic structures etc. He published several research papers in his research area.

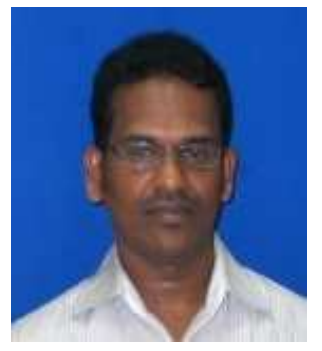

J. Kavikumar received his M.Sc and Ph.D from Annamalai University, India. Since 2006 he has been at Universiti Tun Hussein Onn Malaysia, Malaysia. He has published many papers in national and international journals. His research interests center on the algebra, Fuzzy algebra, Fuzzy functional analysis and Fuzzy numerical analysis.

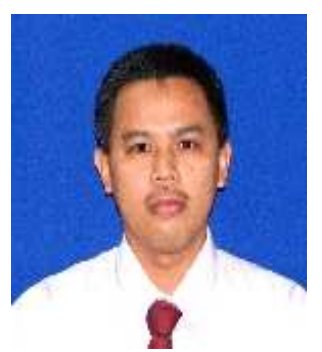

Azme Bin Khamis received his MSc from National University of Malaysia and Ph.D in Statistics from University Technology Malaysia, Malaysia. $\mathrm{He}$ is now the Dean of Faculty of Science, Technology and Human Development at the Universiti Tun Hussein Onn Malaysia, Malaysia. He has published many papers on Neural network and related topics. His research interests focus on the Neural network, Fuzzy Statistics and Fuzzy algebra. 